\title{
Renoprotective Effect of Yiqi Yangyin Huayu Tongluo Formula against Diabetic Nephropathy in Diabetic Rats
}

\author{
Feng-li Wang, ${ }^{1}$ Yue-hua Wang, ${ }^{2}$ Lin Han $\left(D,{ }^{3}\right.$ Hai-yan An, ${ }^{4}$ Jiang-hua Zhang, \\ Xue-yun Zhang, ${ }^{5}$ Zhi-qiang Chen $\left(1,{ }^{6}\right.$ and Jian-guo Qin $\mathbb{1}^{4}$ \\ ${ }^{1}$ Central Laboratory, Dongfang Hospital, Beijing University of Chinese Medicine, Beijing 100078, China \\ ${ }^{2}$ Department of Nephropathy, Hebei Medical University Third Hospital, Shijiazhuang, Hebei 050081, China \\ ${ }^{3}$ School of Basic Medicine, Beijing University of Chinese Medicine, Beijing 100029, China \\ ${ }^{4}$ Department of Nephropathy, Dongfang Hospital, Beijing University of Chinese Medicine, Beijing 100078, China \\ ${ }^{5}$ School of Integrated Traditional Chinese and Western Medicine, Hebei Medical University, Shijiazhuang, Hebei 050017, China \\ ${ }^{6}$ Department of Nephropathy, Hebei Provincial Hospital of Traditional Chinese Medicine, Shijiazhuang, Hebei 050017, China
}

Correspondence should be addressed to Zhi-qiang Chen; chenzhqiang_med@126.com and Jian-guo Qin; qindoctor@163.com

Received 2 July 2018; Revised 30 October 2018; Accepted 15 November 2018; Published 6 December 2018

Academic Editor: Yuewen Gong

Copyright (C) 2018 Feng-li Wang et al. This is an open access article distributed under the Creative Commons Attribution License, which permits unrestricted use, distribution, and reproduction in any medium, provided the original work is properly cited.

Diabetic nephropathy is developed in $20-40 \%$ of patients with diabetes mellitus, and patients with diabetic nephropathy require dialysis and renal transplantation. Traditional Chinese medicine has been widely used in treating patients with diabetic nephropathy in China. However, the detailed mechanisms of traditional Chinese medicine remain unclear. Yiqi Yangyin Huayu Tongluo formula (ZY formula) is a traditional Chinese medicinal formula. Here, we demonstrated kidney protective effect of ZY formula on the rats with diabetic nephropathy. The therapeutic effect of ZY formula on the diabetic nephropathy was almost the same as that of Irbesartan, which proved to have excellent curative effects on diabetic nephropathy. We also demonstrated the mechanism of ZY formula effect on the diabetic nephropathy. First, we validated that the activation of ROS-JNK signaling pathway in diabetic rats could be reduced by ZY. Furthermore, collagen I expression could be downregulated by ZY formula treatment. Meanwhile, cell apoptosis in the kidney of diabetic rats could be alleviated by ZY formula.

\section{Introduction}

Diabetic nephropathy (DN), a leading cause of end-stage renal disease worldwide, is the main microvascular complication of type II diabetes mellitus [1]. DN is developed in $20-40 \%$ of patients with diabetes mellitus, and patients with DN require dialysis and renal transplantation [2]. Thus, clinical strategies for DN prevention will be in urgent need of improving [3]. The pathogenesis of DN is complicated. The characteristic of DN is the persistent albuminuria, accompanied by a progressive elevation of blood pressure, a reduction in glomerular filtration rate, and a reduced risk of cardiovascular events [4]. Both the slowdown of the progression toward end-stage renal disease (ESRD) and the decrease of glomerular filtration rate are closely related to the reduction of albuminuria level. Therefore, the end point for renoprotection could be indicated by the decline in albuminuria [5].

According to the theory of traditional Chinese medicine, it is commonly believed that the pathogenesis of DN is the lack of Yin and Qi. Many Chinese medicine formulas have been reported to be of great clinical efficacy in improving $\mathrm{DN}$, because of the supplement of Yin and Qi from traditional Chinese medicine [6]. The extracellular matrix accumulation of mesangial cells $[7,8]$, signaling pathway in the renal cell $[9$, 10], and diabetes-related glomerular cell apoptosis [11] have widely been reported to be influenced by traditional Chinese medicine. Compared with other Chinese patent medicines available, Yiqi Yangyin Huayu Tongluo formula used in the present study is more innovative and reasonable treatment aimed at the pathogenesis of both Qi and Yin deficiency and blood stasis in DN. 
A hallmark of DN is the extensive accumulation of extracellular matrix (ECM) in the interstitium and glomeruli [12], which leads to tubule-interstitial and glomerulosclerosis fibrosis and basement membrane thickening [13]. Collagen is an important component of ECM. Up to now, 28 types of collagen have been described and identified. The most common types of collagen are type I, type II, type III, type IV, and type $\mathrm{V}$ [14]. The expression of ECM proteins in renal cells is quite different. Type I collagen is expressed in mesangial cells and glomerular epithelial cells, but not glomerular endothelial cells and tubular epithelial cells [15]. During the progression of $\mathrm{DN}$, the components of mesangial matrix, including fibronectin, type VI, V, and IV collagen, as well as other ECM components, such as type II and I collagen, that do not exist in glomeruli in the physiological situation, are extensively accumulated [16]. In particular, the staining study has proved that type IV collagen is increased in patients with DN [17] and the increased urinary excretion of type IV collagen is believed to reflect renal overproduction of this extracellular matrix proteins in early DN [18].

Enhanced oxidative stress is one of the main signaling pathways mediated during DN [19]. The balance between different antioxidant defense systems, including superoxide dismutase, dicarboxylic aldehyde, nicotinamide adenine dinucleotide phosphate, and glutathione peroxidase, determines the extent of oxidative stress $[20,21]$. TGF- $\beta$ expression is upregulated by ROS via MAPK signaling pathway and the nuclear receptor PPAR- $\gamma[22,23]$. Many antioxidant treatments have been reported to be of great therapeutic effect on $\mathrm{DN}[24,25]$.

Renal cell apoptosis, which leads to the functional regression of the kidney, is closely related to the progression of DN [26]. Caspase-3 is the major mediator of cell apoptosis and the activation of caspase- 3 is mediated by proteolytic cleavage of its prodomain [27]. The mitochondrial apoptosis pathway in renal cells is always activated by hyperglycemia and the induced levels of cleaved caspase- 3 can be examined in the kidney of rats with DN [28].

Here, we found that the therapeutic effect of ZY formula to reduce injury in the STZ-induced DN model is almost the same as that of Irbesartan. The renal injury caused by DN can be alleviated by ZY formula through downregulating the level of ROS, ECM accumulation, and apoptosis in the kidney

\section{Materials and Methods}

2.1. Experimental Animals. A total of 40 healthy male SD rats (body weight 120-150g, 4-5 weeks) were supplied by Laboratory Animal Center of Hebei province (Certificate No. 1008095). All experimental procedures were approved by the Ethnic Committee of Hebei Medical University.

2.2. Pharmacological Agents. ZY formula is composed of eight different Chinese herbs, namely, Astragalus membranaceus (2g), Rehmannia (2g), Salvia miltiorrhiza (1.8g), Ligusticum wallichii (1.3g), Rhizoma polygonati (3g), Lumbricus (1g), Leech (1.5g), Buthus martensi kirsch (1g), which were all gifted by Yifang Company (Guangdong, China). The mixed mass ratio for the above eight Chinese herbs was 4:4:3.6:2.6:3:1:1.5:1 and then water was added to adjust the final concentration to $0.3 \mathrm{~g} / \mathrm{ml}$. The dosage of each rat was given according to the human equivalent dose (HED), $d_{B}=$ $\mathrm{d}_{\mathrm{A}} \times \mathrm{R}_{\mathrm{B}} \times \mathrm{W}_{\mathrm{A}}^{1 / 3} \div\left(\mathrm{R}_{\mathrm{A}} \times \mathrm{W}_{\mathrm{B}}^{1 / 3}\right)$, where $\mathrm{d}_{\mathrm{B}}$ is dosage for rat, $\mathrm{d}_{\mathrm{A}}$ is dosage for adults human $(1 \mathrm{~g} / \mathrm{Kg}), R_{B}$ is coefficient of rat body type (0.09), $\mathrm{W}_{\mathrm{A}}$ is body weight of adult human $(60 \mathrm{Kg}), \mathrm{R}_{\mathrm{A}}$ is coefficient of human body type (0.11), and $\mathrm{W}_{\mathrm{B}}$ is body weight of rat. The quality of $\mathrm{ZY}$ formula was controlled according to the Chinese medicine prescription granule fingerprint map. Our previous study has shown that ZY formula has a good clinical effect on early DN [29]. The positive agent, Irbesartan, was purchased from Sanofi Aventis Pharma Ltd (Hangzhou, China). Irbesartan was dissolved in saline solution.

2.3. Establishment of DN Model and Treatment. Before our experiments, totally $40 \mathrm{SD}$ rats were fed at least one week for adaption. Next, the rats in the normal group were fed with a regular diet and other 30 rats received a high-fat diet (Experimental Animal Center of Hebei Medical University, Hebei, China) for 12 weeks. The high-fat diet contains $18.6 \%$ protein, $24.3 \%$ fat, $5.4 \%$ ash, $2.5 \%$ fiber, $6.5 \%$ moisture, and $45.0 \%$ carbohydrate, and the caloric content of food was $4.36 \mathrm{kcal} / \mathrm{g}$. Then these 30 rats were intraperitoneally injected of streptozocin (STZ) at $30 \mathrm{mg} / \mathrm{kg}$, but the rats of normal control group were injected with $0.1 \mathrm{M}$ natrium citricum buffer solution $(\mathrm{pH}=4.5)$ of the same volume. $72 \mathrm{~h}$ after the injection, serum glucose level was detected in all rats. When the serum glucose level was $16.7 \mathrm{mmol} / \mathrm{L}$, the diabetic rat model was successfully established. Then, diabetic rats were divided into the model group, Irbesartan group, and ZY group randomly, with 10 rats in each group. Rats injected with saline solution were considered as the model group $(n=10)$. Rats treated with Irbesartan solution $(11.51 \mathrm{mg} / \mathrm{kg} /$ day) were considered as the Irbesartan group. Rats of $\mathrm{ZY}$ group were treated with ZY formula ( $\mathrm{lg} / \mathrm{kg} / \mathrm{day})$. Rats in different groups were observed for 32 weeks. 32 weeks later, all rats were sacrificed.

2.4. Histopathological Examination. $10 \%$ neutral formalin phosphate buffer was used to fix the renal tissues. Next, the renal tissues were dehydrated through alcohol, embedded into the paraffin, cut into $2 \mu \mathrm{m}$ sections, and stained with hematoxylin-eosin (HE), periodic acid-Schiff (PAS), and Masson. All the sections were examined by light microscopy. Renal tissues of rats were cut into $1 \mathrm{~mm}$ cubes and then fixed in $4 \%$ glutaraldehyde for an hour. Next, the samples were fixed in $1 \%$ osmium tetroxide and dehydrated by graded alcohol series. The samples were embedded in Epon, cut with ultramicrotome (Leica, German), and stained with lead citrate and uranyl acetate. The sections were examined with a Hitachi H-7500 transmission electron microscope. The degree of glomerulosclerosis was calculated by glomerulosclerosis index (GSI), defining that glomeruli (at a magnification of $\times 400$ ) in each kidney was graded in accordance with their severity of glomerular damage using a similar scoring system (0-4 grades) as described previously [30]. And the renal interstitial fibrosis index (RIFI) 
was calculated semiquantitatively according to the ratio of positive area to the area of glomeruli ( 0 , negative; 1 , positive $<10 \%$; 2 , positive $10 \% \sim 25 \%$; 3 , positive $25 \% \sim 50 \%$; and 4 , positive $>50 \%$ ).

2.5. Quantitative Real-Time Polymerase Chain Reaction Anal$y$ sis. Primers used for detection of mRNA were as follows: collagen I: forward $5^{\prime}$ - TCCTGCCGATGTCGCTATC- $3^{\prime}$ and reverse $5^{\prime}$-CCATGTAGGCTACGCTGTTCTTG3'; GAPDH: forward $5^{\prime}$-GTTACCAGGGCTGCCTTCTC-3' and reverse $5^{\prime}$-GGGTTTCCCGTTGATGACC- $3^{\prime}$; caspase-3: forward $5^{\prime}$-GGAGCTTGGAACGCGAAGAA- $3^{\prime}$ and reverse $5^{\prime}$-ACACAAGCCCATTTCAGGGT- $3^{\prime}$. Renal cortical tissues were collected carefully and were frozen at $-80^{\circ} \mathrm{C}$ for extraction of total RNA by Trizol reagent (Beibo Biotechnology Company, Shanghai, China). Next, cDNA was synthesized from total RNA using the SuperScript ${ }^{\mathrm{TM}}$ III First-Strand Synthesis SuperMix for qRT-PCR (Invitrogen, USA) according to the instructions of the manufacturer. Expression of genes was analyzed by quantitative real-time PCR (qPCR). qPCR reaction mixture contained $10 \mu \mathrm{L}$ qPCR mix, $1 \mu \mathrm{L} 10 \mu \mathrm{M}$ forward and reverse primers, and $2 \mu \mathrm{L} c \mathrm{cNA}$, and the volume was brought to $20 \mu \mathrm{L}$ with RNase-free water. The reaction condition was optimized so as to pre-denature at $95^{\circ} \mathrm{C}$ for $2 \mathrm{~min}$, denature at $94^{\circ} \mathrm{C}$ for $20 \mathrm{~s}$, anneal at $60^{\circ} \mathrm{C}$ for $20 \mathrm{~s}$, and extend at $72^{\circ} \mathrm{C}$ for $30 \mathrm{~s}$ for 40 cycles. The RNA quantity was normalized against the GAPDH content, and gene expression was quantified according to the $2^{-\triangle \mathrm{Ct}}$ method.

2.6. Flow Cytometry Analysis of ROS. For exogenous ROS study, single cells of renal tissues were suspended in ice-cold PBS buffer. ROS was measured by staining the cells (Beyotime Biotechnology Company, Jiangsu, China) according to the manufacturer's protocol. After staining, cells were strained briefly and analyzed using Epics-XL II (Beckman-Coulter, USA). The intensity of ROS production by each individual cell was represented by mean fluorescence intensity (MFI).

2.7. Western Blot Analysis. Renal cortical tissues were collected carefully and were frozen at $-80^{\circ} \mathrm{C}$. Proteins for Western blot were extracted by lysing with RIPA buffer from the renal cortex. After centrifugation, the supernatant was collected. Proteins $(35 \mu \mathrm{g})$ were resolved in $12 \%$ sodium dodecyl sulfate polyacrylamide gel (SDS-PAGE) and transferred to the PVDF membrane. The anti-JNK antibody, anti-p-JNK antibody, anti- $\beta$-actin antibody, and anti-caspase- 3 antibody were all purchased from Santa Cruz (California, America). $\beta$-actin was used as the loading control.

2.8. Statistical Analysis. All the data in this study were shown as mean \pm SD and were analyzed by using one-way ANOVA analysis in the SPSS 13.0 (SPSS Inc., USA). The differences between the two groups were analyzed by using SNK assay. The differences were considered statistically significant only when the value of $P$ was less than 0.05 .

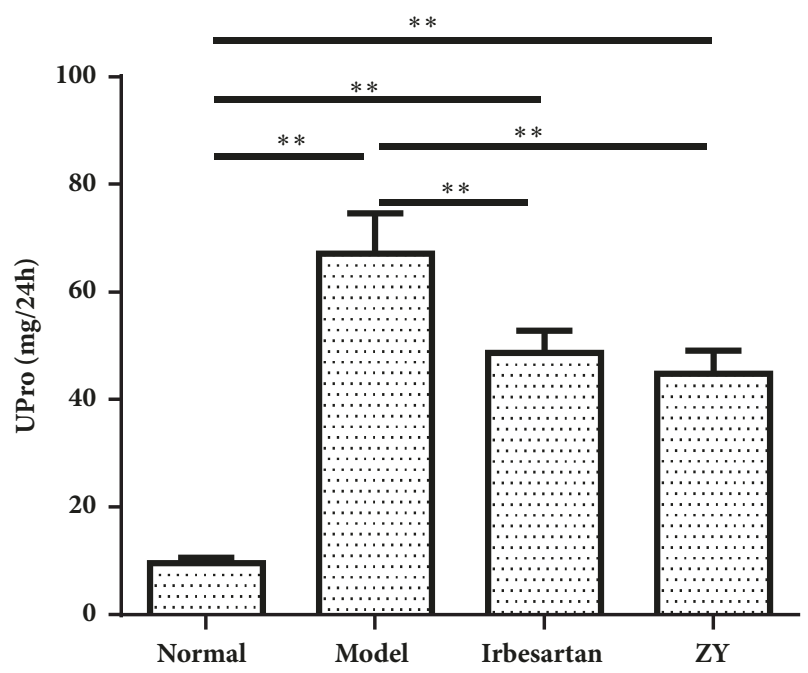

FIGURE 1: Level of urinary protein is reduced by ZY treatment. Changes in the level of urinary protein after treatment. Data were expressed as mean values $( \pm S D)$ from 10 rats. Normal, SD rats; Model, STZ (30mg $/ \mathrm{kg}$ ) treated group; Irbesartan, STZ + Irbesartan $(11.51 \mathrm{mg} / \mathrm{kg})$ treated group; ZY, STZ $+\mathrm{ZY}(1.0 \mathrm{~g} / \mathrm{kg})$ treated group. $* \mathrm{p}<0.05, * * \mathrm{p}<0.01$.

\section{Results}

3.1. The Level of Urinary Protein is Reduced by ZY Formula Treatment. To validate whether the $\mathrm{DN}$ of rats could be induced by STZ and the effect of ZY formula on the regulation of urinary protein, we first examined the level of urinary protein of all the rats. The level of urinary protein of rats with DN induced by STZ was much higher than those of normal rats over the 13-week experimental period (Figure 1). The elevated levels of urinary protein were significantly reduced by ZY formula and Irbesartan treatment from 32 weeks onwards at the doses of $1 \mathrm{~g}$ and $11.51 \mathrm{mg} / \mathrm{kg} \mathrm{BW/} \mathrm{day.} \mathrm{The}$ progression of DN could be slowed down by ZY formula and Irbesartan. Irbesartan had been reported to be of great therapeutic effect on DN. Those rats treated with Irbesartan were set to the positive control. The level of urinary protein between the ZY group and Irbesartan group did not show significant difference (Figure 1). This meant the effect of ZY formula and Irbesartan in reducing urinary protein level of diabetic rats was similar.

3.2. Renal Ultrastructural Changes in Rats with DN Can Be Reversed by ZY Treatment. Next, we examined the histological changes in the kidney of rats. Light microscope was used to examine the histology of kidney in the rats with DN. In $\mathrm{HE}$ stains, there were no obvious abnormalities in glomerular and tubular structures of the kidneys in rats of normal group. However, there were significant mesangial expansion, tubular dilatation, and slightly glomerular hypertrophy in rats of DN model group. However, both after 32 weeks of ZY formula and Irbesartan treatment, the changes in both glomerular and tubular structures induced by STZ were significantly reversed, compared with that of saline treated rats (Figure 2(a)). PAS staining clearly revealed thickening 
A

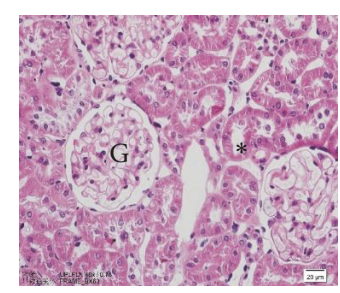

C

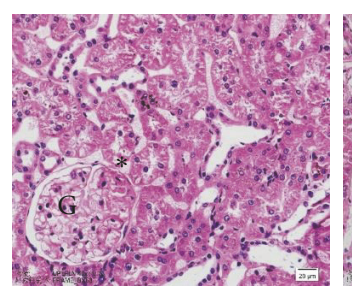

(a)

A

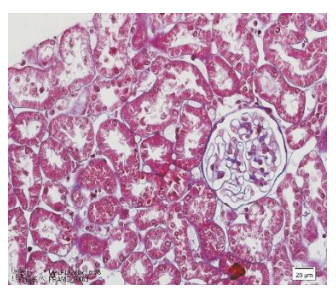

C

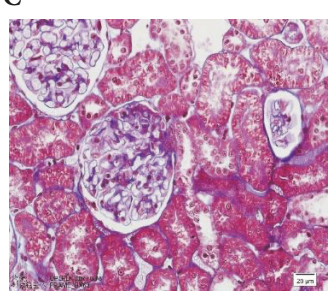

B

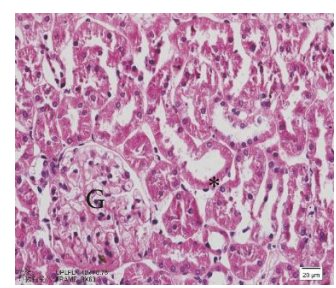

D

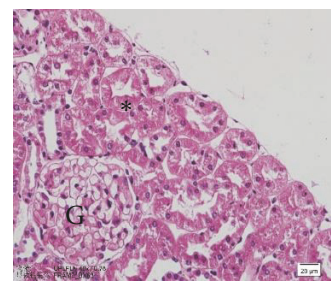

B

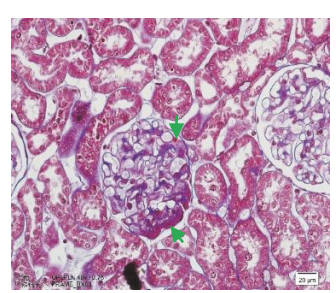

D

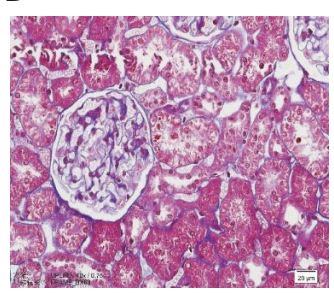

(c)
A

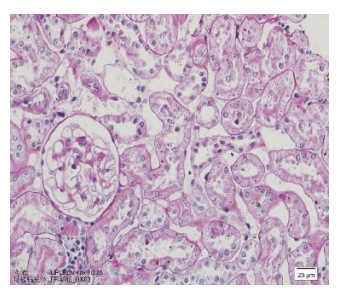

C

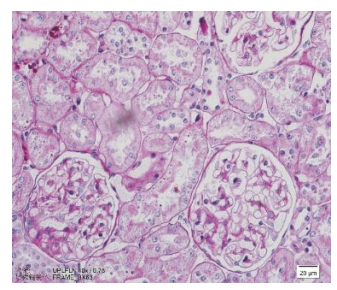

(b)

A

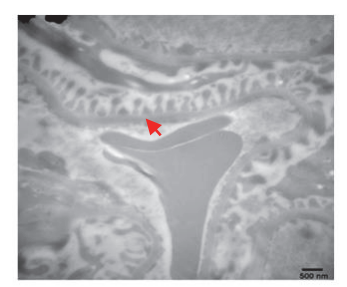

C

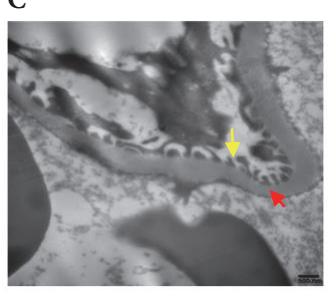

B

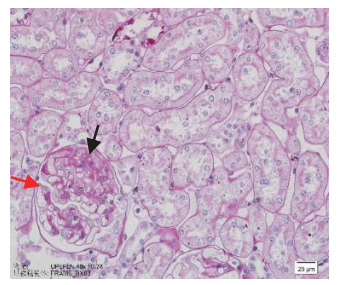

D

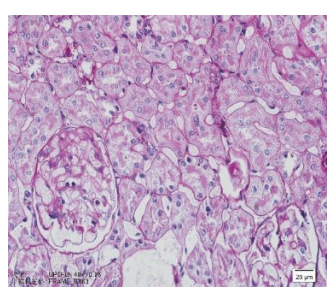

(b)

B

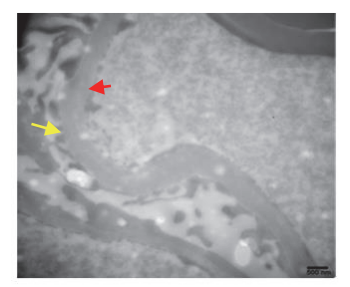

D

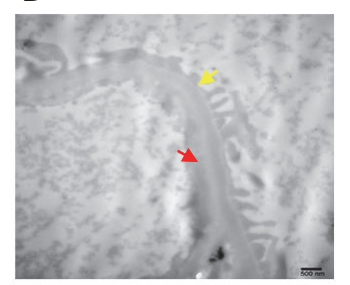

(d)

FIGURE 2: Renal ultrastructural changes in rats with DN can be reversed by ZY treatment. Renal pathological changes in rats with DN after treatment. (a-c) Effects of ZY formula on renal pathology changes in diabetic rats, as assessed by HE staining (400× magnification), PAS staining (400× magnification), and Masson staining (400× magnification). (d) Morphology change in the kidney (transmission electron microscopy. Magnification: 20,000×). Representative microphotographs of the kidney are shown for the normal group (A); model group (B); Irbesartan group (C); and the ZY group (D). G: glomerulus. *: renal tubule. Black arrow: mesangial matrix. Red arrow: glomerular basement membranes (GBM). Green arrow: fibrosis. Yellow arrow: foot process.

of glomerular basement membranes (GBM) and increase of mesangial matrix as compared with controls, which were reversed by ZY formula and Irbesartan treatment (Figure 2(b) and Table 1). Masson trichrome showed increased intensity of fibrosis, indicating accumulation of extracellular matrix proteins (Figure 2(c) and Table 1). Next, the morphometric differences between different groups were analyzed by electron microscope. Compared with the normal rats, significant thickness of GBM, fusion of foot process accompanied with lager width of foot process, was observed in the rats with DN. After the rats with DN were treated with ZY formula and Irbesartan, the changes in the fusion of foot process can be reversed significantly but without significant change in GBM (Figure 2(d)). These results suggest that the histological changes induced by $\mathrm{DN}$ can be reversed by ZY formula and Irbesartan.

3.3. The ROS-JNK Signaling Pathway in the Kidney. ROS production in the kidney was always induced by high blood glucose. Exposure to the STZ, the ROS production in the kidney of rats, was significantly upregulated. However, ZY formula and Irbesartan partially reduced STZ-induced ROS production. No significant difference was seen on the level of ROS production between ZY and Irbesartan treatment (Figure 3(a)).

MAPK signaling pathway could be activated by ROS. JNKs belonged to the MAPK family and played an important role in the MAPK pathway. The JNK gene expression 


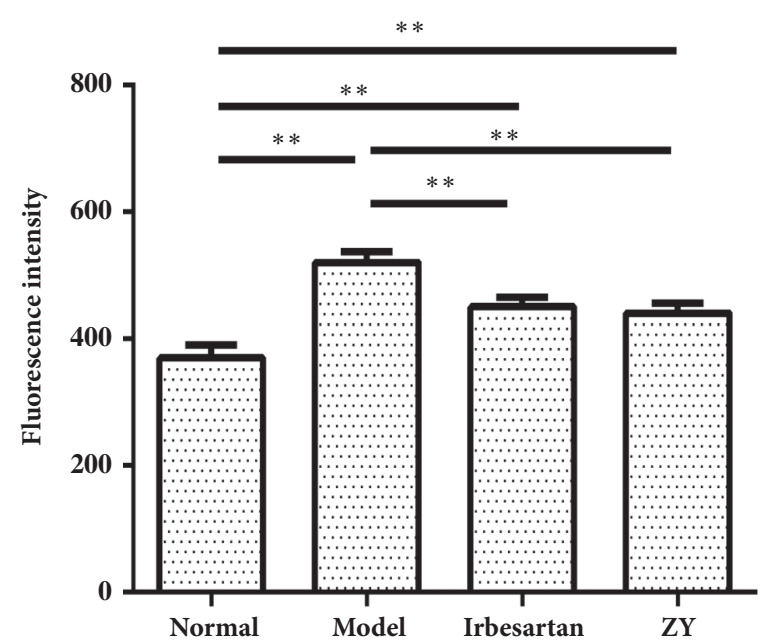

(a)
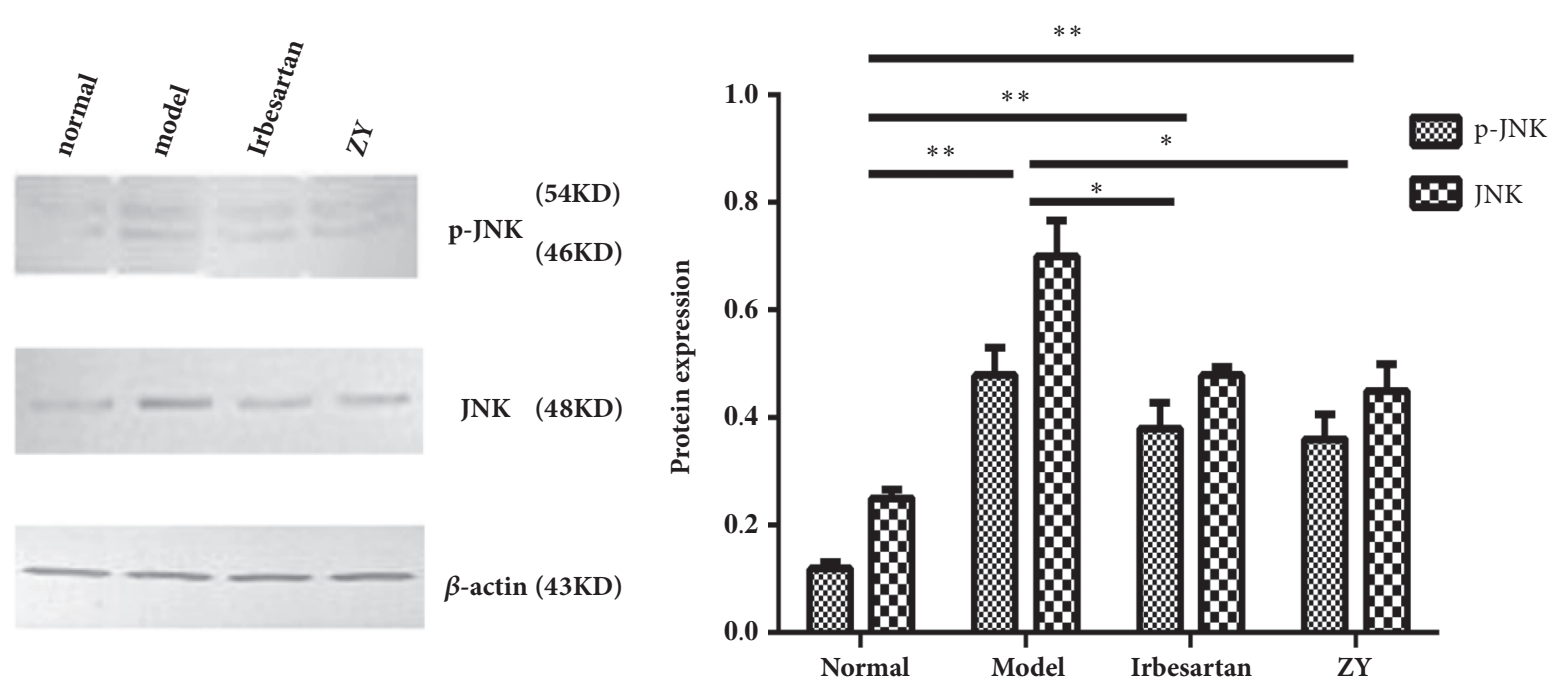

(b)

FIGURE 3: The ROS-JNK signaling pathway in the kidney. Effects of ZY formula and Irbesartan on ROS-JNK signaling pathway in DN model rats. (a) Effects of ZY formula and Irbesartan on ROS level in the kidney. (b) Effects of ZY formula and Irbesartan on the expression of JNK and phosphorylated JNK in the kidney. The Western blot results were presented on the left and the semiquantitative results determined by $\mathrm{WB}$ were presented on the right. $\mathrm{SD}$ rats were divided into normal group, model group, Irbesartan group, and $\mathrm{ZY}$ group. $* \mathrm{p}<0.05, * * \mathrm{p}<0.01$.

TABLE 1: Quantification of PAS (GSI) and Masson (RIFI) staining.

\begin{tabular}{lcc}
\hline Group & GSI & RIFI \\
\hline Normal group & $0.12 \pm 0.01$ & $0.13 \pm 0.01$ \\
Model group & $1.20 \pm 0.04^{\mathrm{a}}$ & $0.53 \pm 0.03^{\mathrm{a}}$ \\
Irbesartan group & $0.87 \pm 0.04^{\mathrm{ab}}$ & $0.37 \pm 0.02^{\mathrm{ab}}$ \\
ZY group & $0.81 \pm 0.03^{\mathrm{ab}}$ & $0.35 \pm 0.03^{\mathrm{ab}}$ \\
\hline
\end{tabular}

${ }^{a} \mathrm{p}<0.05$ vs. normal group

${ }_{\mathrm{p}} \mathrm{p}<0.05$ vs. model group

${ }^{c} \mathrm{p}<0.05$ vs. Irbesartan group 


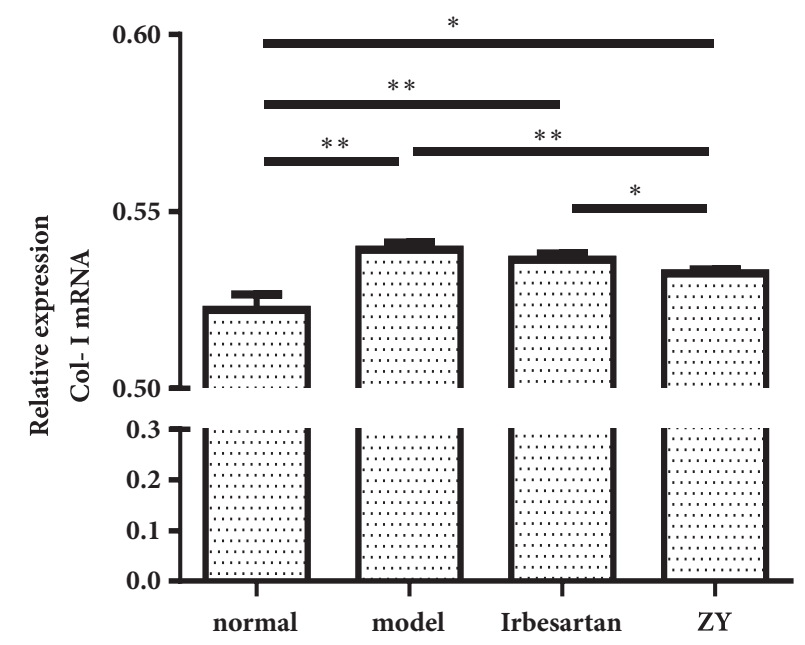

FIGURE 4: Effect of ZY treatment on collagen I expression. Effects of ZY formula and Irbesartan on the expression of collagen I in DN model rats. The mRNA level of collagen I in the kidney was examined by real-time PCR. SD rats were divided into normal group, model group, Irbesartan group, and $\mathrm{ZY}$ group. $* \mathrm{p}<0.05$, $* * \mathrm{p}<0.01$.

correlated greatly with the development of type II diabetes $[31,32]$. According to the Western blot analysis, the levels of both JNK and phosphorylated JNK proteins in the kidney of rats with DN were higher than in the kidney of normal rats. Treatment of DN with either ZY formula or Irbesartan significantly reduced the diabetic activation of JNK (the level of phosphorylated JNK) (Figure 3(b)). These results suggested that ROS and JNK pathway could be induced in the rats with $\mathrm{DN}$, but $\mathrm{ZY}$ formula could suppress the diabetic activation of both ROS and JNK pathway.

3.4. Effect of ZY Formula on Collagen I Expression. We next wanted to examine whether ZY formula had an effect on the accumulation of extracellular matrix (ECM). The results showed that the mRNA level of collagen I was higher in the diabetic model group, compared with the normal group. After treatment with ZY formula, the expression of collagen I obviously decreased, compared with the diabetic model group and Irbesartan group (Figure 4). This result suggested that activation of collagen I expression in the rats with DN could be reversed by ZY formula treatment.

3.5. ZY Protected Kidney of Diabetic Rats from Apoptosis. To determine the antiapoptotic effect of ZY formula, flow cytometry assay was performed. The apoptosis rate of renal cortex cells was significantly increased compared with normal rats. The treatment of both ZY formula and Irbesartan was effective in reducing apoptosis in diabetic rats. There was no significant difference between the ZY group and Irbesartan group (Figure 5(a)).

The activation of caspase- 3 was closely related to the activation of apoptosis pathway. In diabetic rats induced by STZ, the quantitative real-time PCR examination (Figure 5(b)) and immunohistochemically staining (Figure 5(c)
TABLE 2: Quantification of caspase-3 staining.

\begin{tabular}{lc}
\hline Group & Percentage area (\%) \\
\hline Normal group & $4.53 \pm 0.44$ \\
Model group & $13.72 \pm 0.38^{\mathrm{a}}$ \\
Irbesartan group & $7.91 \pm 0.35^{\mathrm{ab}}$ \\
ZY group & $8.11 \pm 0.41^{\mathrm{ab}}$ \\
\hline${ }^{\mathrm{a}} \mathrm{p}<0.05$ vs. normal group & \\
${ }^{\mathrm{b}} \mathrm{p}<0.05$ vs. model group & \\
${ }^{\mathrm{c}}<0.05$ vs. Irbesartan group & \\
\end{tabular}

and Table 2) for caspase-3 revealed that strong PCR signaling and immunoreactivity of caspase- 3 were detected in the kidney. The caspase-3 expression in the kidney was restored in diabetic rats treated with ZY formula. Next, the cleaved caspase-3 was also detected by Western blot (Figure 5(d)). These results showed that the active forms of caspase- 3 were increased in the kidney of rats with $\mathrm{DN}$, but could be restored after treatment with ZY formula and Irbesartan. These results suggested that renal cell apoptosis induced by STZ could be reduced by $\mathrm{ZY}$ formula treatment.

\section{Discussion}

$\mathrm{DN}$ is a kind of progressive disease that causes injury of kidney, with progression over time [33]. In the early stage of $\mathrm{DN}$, there are some pathological changes in the kidney that can be examined, such as mesangial matrix accumulation, enhanced oxidative stress, and renal cell apoptosis. In our study, we found that ZY formula had a great effect in reducing the ECM accumulation, ROS, and cell apoptosis in the kidney, which means that early syndrome of $\mathrm{DN}$ could be alleviated and the progression of $\mathrm{DN}$ could be inhibited by $\mathrm{ZY}$ formula.

The TGF- $\beta$ signaling pathway is believed to contribute to a predominant role in the progression of DN. Most of the traditional Chinese medicine or medicinal formulas have a great effect on the TGF- $\beta$ signaling pathway. Tangshen formula protected the kidney of diabetic rats from diabetic injuries by reducing the expression of TGF- $\beta$ receptor I and TGF- $\beta$ [34]. The renal injury in diabetic rats could be reduced significantly by She Jing Xiao Bai capsule and the mechanism involved was the decrease of CTGF and TGF- $\beta$ expression induced by She Jing Xiao Bai capsule treatment [35]. In addition, the lipid metabolism and TGF- $\beta$ signaling pathway could be affected by Bebeerine [36] and Gui Qi mixture [37], which led to the recovery of kidney in DN rats. However, in our study, we found that the JNK signaling pathway was affected by ZY formula. After the rats were induced by the STZ, the JNK signaling pathway in the kidney was activated. When the diabetic rats were treated with the ZY formula, the activation of JNK signaling pathway could be reduced in the kidney. Most of the traditional Chinese medicinal formulas consisted of different herbs in order to improve the therapeutic efficacy and reduce side effects. Thus, many signaling pathways can be affected by these traditional Chinese medicinal formulas. Due to the important role of TGF- $\beta$ signaling pathway and the multiple targets of traditional Chinese medicine, the influence of $\mathrm{ZY}$ formula 
(a)

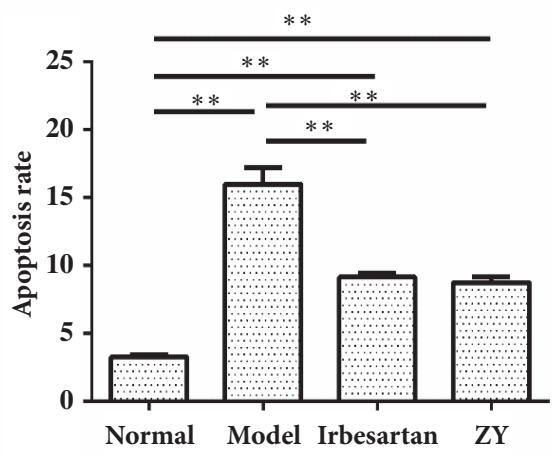

(b)

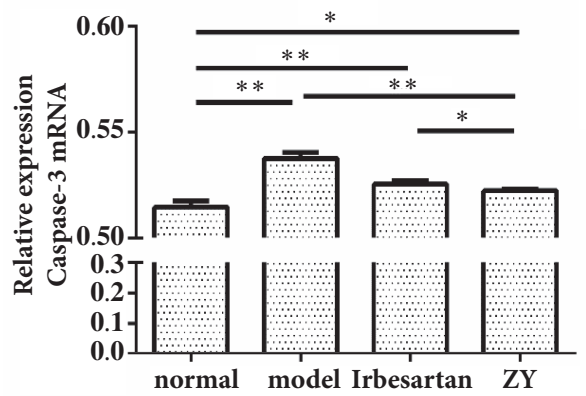

(d)

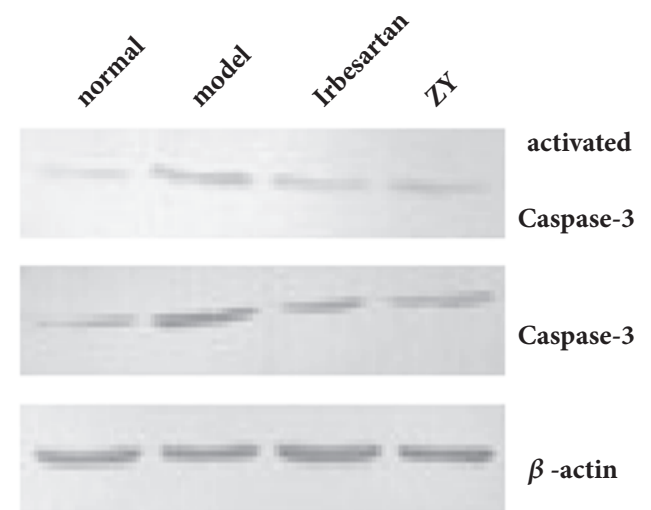

(c)

A

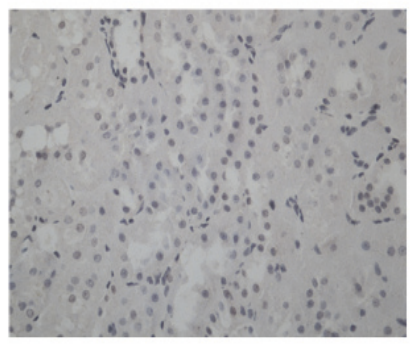

C

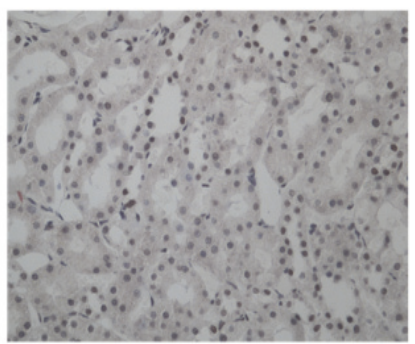

B

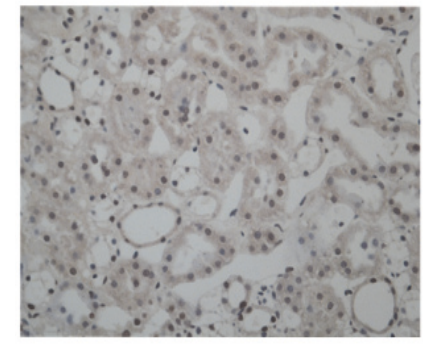

D

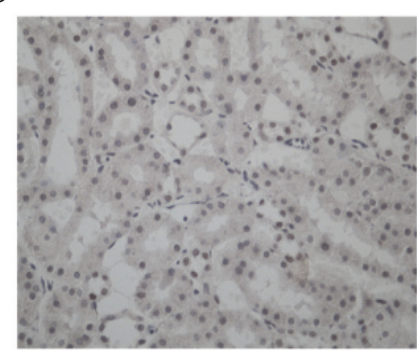

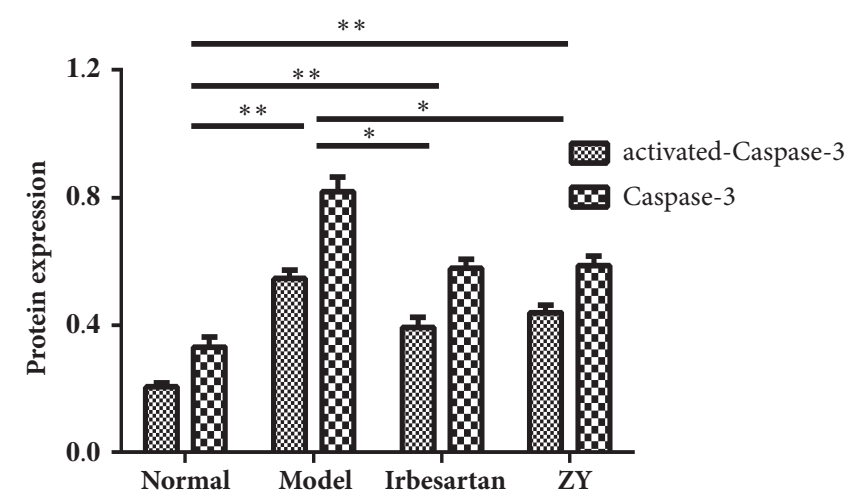

FIGURE 5: ZY protected kidney of diabetic rats from apoptosis. ZY formula protected kidney of diabetic rats from apoptosis. (a) The level of renal cells apoptosis was measured by flow cytometry assay and the data were expressed as mean $\pm \mathrm{SD}$. $* \mathrm{p}<0.05, * * \mathrm{p}<0.01$. (b) The mRNA level of caspase- 3 was examined by real-time PCR. The data were expressed as mean \pm SD. $* \mathrm{p}<0.05, * * \mathrm{p}<0.01$. (c) The expression of caspase- 3 in the kidney was examined by immunohistochemically staining (400× magnification). (d) Western blot was used to examine the expression of caspase- 3 and cleaved caspase-3. The Western blot results were presented on the left and the semiquantitative results determined by WB were presented on the right. $* \mathrm{p}<0.05, * * \mathrm{p}<0.01$. SD rats were divided into normal group, model group, Irbesartan group, and $\mathrm{ZY}$ group.

on TGF- $\beta$ signaling pathway or other signaling pathways needs to be further examined. Also, in our future work, the major active components of $\mathrm{ZY}$ formula responsible for the renoprotection should be identified.

The accumulation of extracellular matrix (ECM) in glomeruli is one of the most remarkable hallmarks of DN [38]. Type IV collagen is the major component of the basement membranes of kidney [39]. Some of the traditional Chinese medicines, such as Tangshen formula [34] and Danggui-Shaoyao-San [33], protected kidney from renal injury through regulating the accumulation of extracellular matrix in glomeruli. Although the upregulation of type IV collagen expression is the main cause of ECM in glomeruli [40], the roles of type I collagen during the progression 
of DN should not be neglected. Type I collagen does not exist in glomeruli in the physiological situation. But during the progression of DN, type I collagen starts to accumulate in glomeruli which results in the renal fibrosis and renal function loss [16]. Type I collagen seems to be a biomarker of DN progression. In our study, we found that type I collagen expression could be affected by ZY formula treatment. But the accumulation of ECM in glomeruli is caused not only by the production of ECM associated protein, but also by the inhibition of ECM associated protein degradation. How ZY formula affects the ECM metabolism needs further examinations.

Reactive oxygen species (ROS) release in the kidney was one of the characteristics of DN progression [41]. The ROS generation and oxidative modified proteins production were increased in mitochondria of renal tubule cells of rats after the induction of STZ [42]. In the kidney of rats with DN, the SOD activity was decreased and the MDA level was upregulated [43]. ROS generation in the kidney could be inhibited by Curcuma aromatic [44]. The SOD activity was increased and MDA level was reduced after the treatment of Fufang Xue Shuan Tong capsules [45], Crocin [46], and Luteolin [47] in diabetic rats and then inhibited the progression of $\mathrm{DN}$. We found that the ROS generation in diabetic rats could be inhibited by ZY formula. The process of ROS generation in the kidney is complicated, so the mechanism of ZY formula to affect ROS production needs to be further examined.

\section{Conclusion}

The therapeutic effect of ZY formula to reduce renal injury in the STZ-induced DN model was almost the same as that of Irbesartan. The renal injury caused by DN could be alleviated by ZY formula through downregulating the ROS generation, ECM accumulation, and renal cells apoptosis in the kidney.

\section{Data Availability}

No data were used to support this study.

\section{Conflicts of Interest}

The authors declare that there are no conflicts of interest associated with the paper.

\section{Acknowledgments}

This work was supported by the National Natural Science Foundation of China (No. 81173419).

\section{References}

[1] J. F. Navarro-González, C. Mora-Fernández, M. M. de Fuentes, and J. García-Pérez, "Inflammatory molecules and pathways in the pathogenesis of diabetic nephropathy," Nature Reviews Nephrology, vol. 7, no. 6, pp. 327-340, 2011.
[2] A. J. Collins, R. N. Foley, and B. Chavers, "United States Renal Data System 2011 Annual Data Report: Atlas of chronic kidney disease \& end-stage renal disease in the United States," in American journal of kidney diseases: the official journal of the National Kidney Foundation, vol. 59, supplement 1, p. A7, 2012.

[3] H.-H. Parving, F. Persson, J. B. Lewis, E. J. Lewis, and N. K. Hollenberg, "Aliskiren combined with losartan in type 2 diabetes and nephropathy," The New England Journal of Medicine, vol. 358, no. 23, pp. 2433-2446, 2008.

[4] B. Brenner, M. Cooper, and D. de Zeeuw, "Effects of losartan on renal and cardiovascular outcomes in patients with type 2 diabetes and nephropathy," ACC Current Journal Review, vol. 11, no. 1, p. 26, 2002.

[5] E. Lewis, L. Hunsicker, and W. Clarkeet al, "Renoprotective effects of the angiotensin-receptor antagonist irbesartan in patients with nephropathy due to type 2 diabetes," ACC Current Journal Review, vol. 11, no. 1, p. 26, 2002.

[6] X.-L. Tong, L. Dong, L. Chen, and Z. Zhen, "Treatment of diabetes using traditional Chinese medicine: past, present and future," American Journal of Chinese Medicine, vol. 40, no. 5, pp. 877-886, 2012.

[7] Y.-G. Du, K.-N. Zhang, Z.-L. Gao, F. Dai, X.-X. Wu, and K.F. Chai, "Tangshen formula improves inflammation in renal tissue of diabetic nephropathy through SIRT1/NF- $\kappa$ B pathway," Experimental and Therapeutic Medicine, vol. 15, no. 2, pp. $2156-$ 2164, 2018.

[8] X. H. Li, G. P. Lei, and J. T. Li, "Effect of TongluoYishen formula on rearrangement of cytoskeleton of glomerular podocyte in diabetic nephropathy rats," vol. 10, pp. 8458-8463, 2017.

[9] N. Zhang, Y. B. Gao, D. W. Zou, and etal., "Effects of Chinese Medicine Tong xinluo on Diabetic Nephropathy via Inhibiting TGF-beta 1-Induced Epithelial-to-Mesenchymal Transition," Evidence-Based Complementary and Alternative Medicine, Article ID 123497, 2014.

[10] H. Liu, J. Zheng, and R.-H. Li, "Clinical efficacy of 'Spleenkidney-care' Yiqi Huayu and Jiangzhuo traditional Chinese medicine for the treatment of patients with diabetic nephropathy," Experimental and Therapeutic Medicine, vol. 10, no. 3, pp. 1096-1102, 2015.

[11] L. Zhou, X.-F. An, S.-C. Teng et al., "Pretreatment with the total flavone glycosides of Flos Abelmoschus manihot and hyperoside prevents glomerular podocyte apoptosis in streptozotocininduced diabetic nephropathy," Journal of Medicinal Food, vol. 15, no. 5, pp. 461-468, 2012.

[12] M. Miyazaki, T. Nishino, K. Abe, A. Furusu, T. Koji, and S. Kohno, "Regulation of renal extracellular matrix metabolism," Contributions to Nephrology, vol. 139, pp. 141-155, 2003.

[13] F. P. Schena and L. Gesualdo, "Pathogenetic mechanisms of diabetic nephropathy," Journal of the American Society of Nephrology, vol. 16, no. 3, supplement 1, pp. S30-S33, 2005.

[14] M. A. Karsdal, S. H. Nielsen, D. J. Leeming et al., "The good and the bad collagens of fibrosis - Their role in signaling and organ function," Advanced Drug Delivery Reviews, vol. 121, pp. 43-56, 2017.

[15] M. Miyazaki, M. Endoh, Y. Nomoto et al., "A Sensitive Method of Non-Radioactive In Situ Hybridization for mRNA Localization within Human Renal Biopsy Specimens: Use of Digoxigenin Labeled Oligonucleotides," Internal Medicine, vol. 33, no. 2, pp. 87-91, 1994.

[16] T. Nakamura, I. Ebihara, I. Shirato, Y. Tomino, and H. Koide, "Increased steady-state levels of mRNA coding for extracellular 
matrix components in kidneys of NZB/W F1 mice," The American Journal of Pathology, vol. 139, no. 2, pp. 437-450, 1991.

[17] S. G. Adler, S. Feld, L. Striker et al., "Glomerular type IV collagen in patients with diabetic nephropathy with and without additional glomerular disease," Kidney International, vol. 57, no. 5, pp. 2084-2092, 2000.

[18] M. P. Cohen, C. W. Shearman, and G. T. Lautenslager, "Serum type IV collagen in diabetic patients at risk for nephropathy," Diabetes Care, vol. 24, no. 8, pp. 1324-1327, 2001.

[19] C.-W. Kuo, C.-J. Shen, Y.-T. Tung et al., "Extracellular superoxide dismutase ameliorates streptozotocin-induced rat diabetic nephropathy via inhibiting the ROS/ERK1/2 signaling," Life Sciences, vol. 135, pp. 77-86, 2015.

[20] T. Djordjevic, A. Pogrebniak, R. S. BelAiba et al., “The expression of the NADPH oxidase subunit p22phox is regulated by a redox-sensitive pathway in endothelial cells," Free Radical Biology \& Medicine, vol. 38, no. 5, pp. 616-630, 2005.

[21] S. Chu, L. Wang, X.-D. Mao, and W. Peng, "Improvement of Huangqi Decoction on Endothelial Dysfunction in 5/6 Nephrectomized Rats," Cellular Physiology and Biochemistry, vol. 40, no. 6, pp. 1354-1366, 2016.

[22] H. B. Lee, M. Yu, Y. Yang, Z. Jiang, and H. Ha, "Reactive oxygen species-regulated signaling pathways in diabetic nephropathy," Journal of the American Society of Nephrology, vol. 14, no. 8, supplement 3, pp. S241-S245, 2003.

[23] S. Reuter, S. C. Gupta, M. M. Chaturvedi, and B. B. Aggarwal, "Oxidative stress, inflammation, and cancer: how are they linked?" Free Radical Biology \& Medicine, vol. 49, no. 11, pp. 1603-1616, 2010.

[24] G. Komeili, M. Hashemi, and M. Bameri-Niafar, "Evaluation of antidiabetic and antihyperlipidemic effects of peganum harmala seeds in diabetic rats," Cholesterol, vol. 2016, pp. 1-6, 2016.

[25] K. Carolo Dos Santos, C. Pereira Braga, P. Octavio Barbanera, F. Rodrigues Ferreira Seiva, A. Fernandes Jr., and A. A. Henrique Fernandes, "Cardiac energy metabolism and oxidative stress biomarkers in diabetic rat treated with resveratrol," PLoS ONE, vol. 9, no. 7, Article ID e102775, 2014.

[26] D. Verzola, M. T. Gandolfo, F. Ferrario et al., "Apoptosis in the kidneys of patients with type II diabetic nephropathy," Kidney International, vol. 72, no. 10, pp. 1262-1272, 2007.

[27] M. Whyte, "ICE/CED-3 proteases in apoptosis," Trends in Cell Biology, vol. 6, no. 7, pp. 245-248, 1996.

[28] T. Mohan, P. Velusamy, L. N. Chakrapani et al., "Impact of EGCG Supplementation on the Progression of Diabetic Nephropathy in Rats: An Insight into Fibrosis and Apoptosis," Journal of Agricultural and Food Chemistry, vol. 65, no. 36, pp. 8028-8036, 2017.

[29] F. L. Wang, Z. Q. Chen, and Y. H. Wang, "Clinical observation of treating early diabetic nephropathy by qi supplementing, yin nourishing, blood stasis dispersing, collateral dredging recipe," Chinese Journal of Integrated Traditional \& Western Medicine, vol. 32, no. 1, pp. 35-38, 2012.

[30] H. Zhang, P. Li, F. J. Burczynski, and etal., "Attenuation of Diabetic Nephropathy in Otsuka Long-Evans Tokushima Fatty (OLETF) Rats with a Combination of Chinese Herbs (Tangshen Formula)," Evidence-Based Complementary and Alternative Medicine, vol. 2011, Article ID 613737, 2011.

[31] A. P. Lakshmanan, R. A. Thandavarayan, K. Watanabe et al., "Modulation of AT-1R/MAPK cascade by an olmesartan treatment attenuates diabetic nephropathy in streptozotocininduced diabetic mice," Molecular and Cellular Endocrinology, vol. 348, no. 1, pp. 104-111, 2012.

[32] Y. Wang, Y. Wang, M. Luo et al., "Novel curcumin analog C66 prevents diabetic nephropathy via JNK pathway with the involvement of p300/CBP-mediated histone acetylation," Biochimica et Biophysica Acta (BBA) - Molecular Basis of Disease, vol. 1852, no. 1, pp. 34-46, 2015.

[33] I.-M. Liu, T.-F. Tzeng, S.-S. Liou, and C. J. Chang, "Beneficial effect of traditional chinese medicinal formula DangguiShaoyao-San on advanced glycation end-product-mediated renal injury in streptozotocin-diabetic rats," Evidence-Based Complementary and Alternative Medicine, vol. 2012, Article ID 140103, 10 pages, 2012.

[34] T. Zhao, S. Sun, H. Zhang et al., "Therapeutic effects of tangshen formula on diabetic nephropathy in rats," PLoS ONE, vol. 11, no. 1, Article ID e0147693, 2016.

[35] O. Jin, L. P. Gao, X. Chen, and etal., "Protective effects of She Jing Xiao Bai capsule on diabetic nephropathy in a diabetic rat model," International Journal of Clinical and Experimental Medicine, vol. 9, no. 2, pp. 2873-2880, 2016.

[36] S.-F. Sun, T.-T. Zhao, H.-J. Zhang et al., "Renoprotective effect of berberine on type 2 diabetic nephropathy in rats," Clinical and Experimental Pharmacology and Physiology, vol. 42, no. 6, pp. 662-670, 2015.

[37] Y.-W. Zhang, D. Xie, Y.-X. Chen, H.-Y. Zhang, and Z.-X. $\mathrm{Xia}$, "Protective effect of Gui Qi mixture on the progression of diabetic nephropathy in rats," Experimental and Clinical Endocrinology \& Diabetes, vol. 114, no. 10, pp. 563-568, 2006.

[38] R. J. Falk, J. I. Scheinman, S. M. Mauer, and A. F. Michael, "Polyantigenic expansion of basement membrane constituents in diabetic nephropathy," Diabetes, vol. 32, supplement 2, pp. 34-39, 1983.

[39] A. Tanaka, E. Ohmura, Y. Imai, F. Matsuzaki, S. Kawazu, and H. Horie, "Clinical implications of urinary type 4 collagen for early detection of diabetic nephropathy and retinopathy," Diabetes, vol. 50, pp. A468-A468, 2001.

[40] S. Yung, M. K. M. Chau, Q. Zhang, C. Z. Zhang, and T. M. Chan, "Sulodexide decreases albuminuria and regulates matrix protein accumulation in C57BL/6 mice with streptozotocin induced type I diabetic nephropathy," PLoS ONE, vol. 8, no. 1, Article ID e54501, 2013.

[41] Z. Cao and M. E. Cooper, "Pathogenesis of diabetic nephropathy," Journal of Diabetes Investigation, vol. 2, no. 4, pp. 243-247, 2011.

[42] T. Kanno, E. F. Sato, S. Muranaka et al., "Oxidative stress underlies the mechanism for $\mathrm{Ca} 2+-$ induced permeability transition of mitochondria," Free Radical Research, vol. 38, no. 1, pp. 2735, 2004.

[43] M. Mohora, B. Vîrgolici, F. Paveliu, D. Lixandru, C. Muscurel, and M. Greabu, "Free radical activity in obese patients with type 2 diabetes mellitus.," Romanian journal of internal medicine $=$ Revue roumaine de médecine interne, vol. 44, no. 1, pp. 69-78, 2006.

[44] J.-Y. Hong, E. F. Sato, Y. Kira, M. Nishikawa, K. Shimada, and M. Inoue, "Curcuma aromatica inhibits diabetic nephropathy in the rat," Journal of Food Science, vol. 71, no. 9, pp. S626-S632, 2006.

[45] D. Fang, X. Wan, W. Deng et al., "Fufang Xue Shuan tong capsules inhibit renal oxidative stress markers and indices of nephropathy in diabetic rats," Experimental and Therapeutic Medicine, vol. 4, no. 5, pp. 871-876, 2012. 
[46] H. O. Abou-Hany, H. Atef, E. Said, H. A. Elkashef, and H. A. Salem, "Crocin mediated amelioration of oxidative burden and inflammatory cascade suppresses diabetic nephropathy progression in diabetic rats," Chemico-Biological Interactions, vol. 284, pp. 90-100, 2018.

[47] G. G. Wang, X. H. Lu, W. Li, X. Zhao, and C. Zhang, "Protective effects of luteolin on diabetic nephropathy in STZ-induced diabetic rats," Evidence-Based Complementary and Alternative Medicine, vol. 2011, Article ID 323171, 7 pages, 2011. 


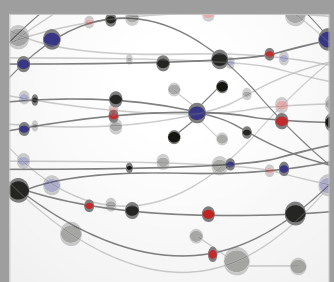

The Scientific World Journal
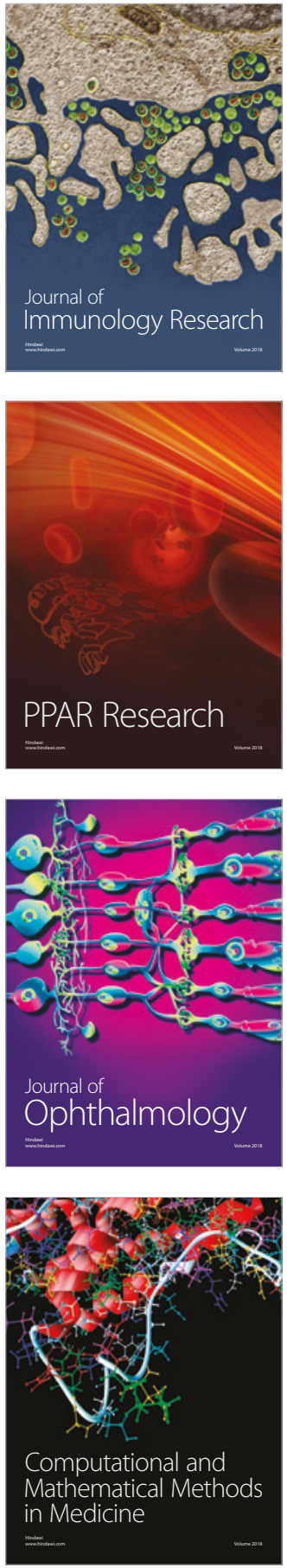

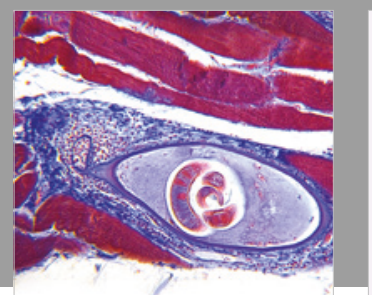

Gastroenterology Research and Practice

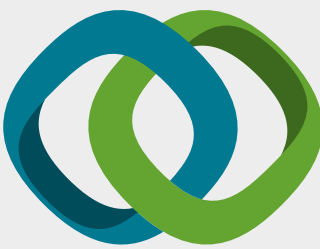

\section{Hindawi}

Submit your manuscripts at

www.hindawi.com
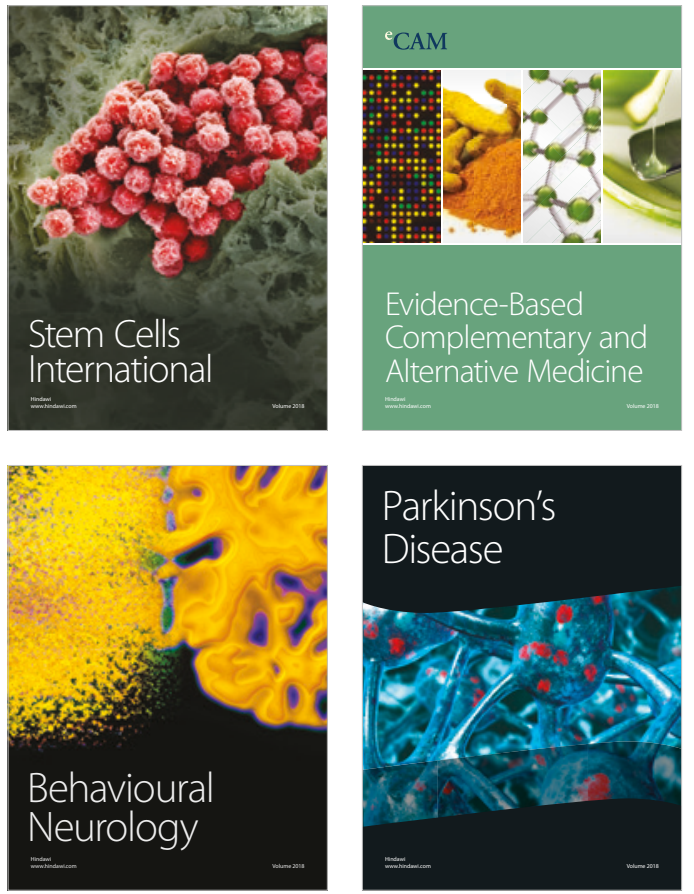

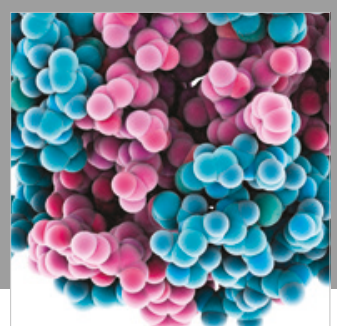

ournal of

Diabetes Research

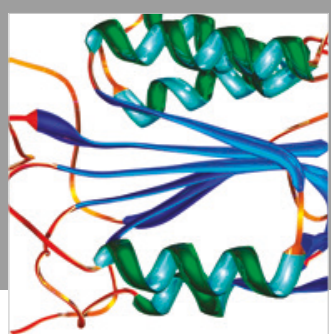

Disease Markers
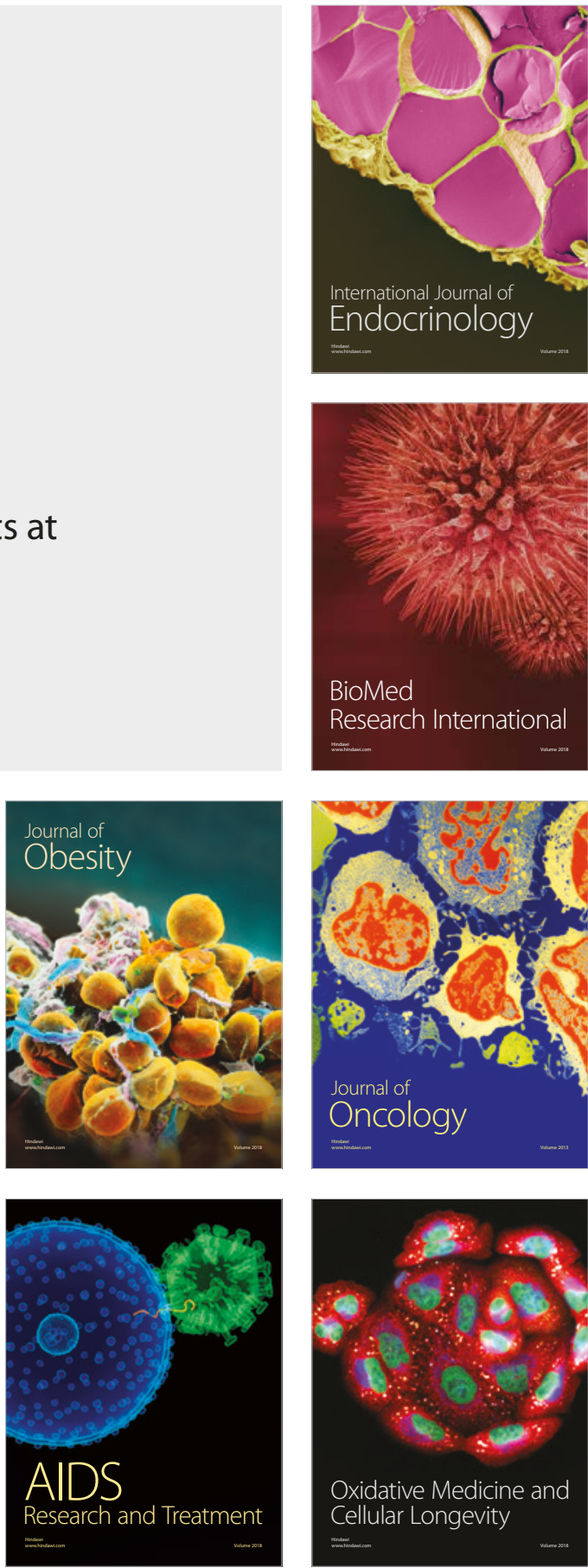